\title{
Association of pre-pregnancy BMI with biochemical profile during pregnancy, delivery mode and size of neonates in the CRIBS birth cohort
}

\author{
Dubravka Havaš Auguštin ${ }^{1}$, Jelena Šrac ${ }^{1}$, Matea Zajc Petranovićc ${ }^{1}$, Natalija Novokmet ${ }^{1}$, \\ Ivan Dolanc $^{1}$, Tonko Caric ${ }^{1}$, Ellen Demerath ${ }^{2}$, Noel Cameron ${ }^{3}$ and Saša Missoni ${ }^{1,4}$ \\ ${ }^{1}$ Institute for Anthropological Research, Zagreb, Croatia, \\ ${ }^{2}$ School of Public Health, University of MinnesotaSchool of Public Health, University of Minnesota, Minneapolis, \\ USA, \\ ${ }^{3}$ School of Sport, Exercise and Health Sciences, Loughborough University, Loughborough, United Kingdom and \\ ${ }^{4} J$. J. Strossmayer" University, School of Medicine, Osijek, Croatia
}

\begin{abstract}
In the last few decades the prevalence of overweight and obesity has reached epidemic levels worldwide affecting over 2.1 billion people, including a large proportion of women of childbearing age. An adverse intrauterine environment resulting from conditions such as maternal obesity or gestational diabetes, can have long-term negative effect on the child's health later in life. This study focuses on maternal pre-pregnancy BMIs and their association with biochemical profile in pregnancy, delivery mode and size of neonates in the Croatian Islands' Birth cohort Study (CRIBS). A total of 301 mother-child dyads were included in this study. The classification of the pre-pregnancy nutritional status followed WHO BMI cut-offs. Blood samples of women were taken in the second trimester of pregnancy and analysed for fasting blood glucose level, urates, creatinine, uric acid, total protein, bilirubin, triglycerides, total cholesterol, HDL and LDL cholesterol, potassium, sodium, aspartate transaminase (AST), alanine aminotransferase (ALT), gamma-glutamyl transferase (GGT), homocysteine, fibrinogen and lipoprotein A. The neonates' length in cm and weight in gm were measured immediately after delivery at the University Hospital Split. Differences in mean values of biochemical parameters and neonates' size between pre-pregnancy BMI categories were tested using One-way ANOVA $(\mathrm{p}<0.05)$ and differences in prevalence of delivery mode using the Chi-square test. Nutritional status before pregnancy showed that $4 \%$ of women were underweight $(\mathrm{BMI}<18.5 \mathrm{~kg}$. $\mathrm{m}^{2}$ ), 75.1\% had normal weight (BMI 18.5-24.9 kg. $\mathrm{m}^{2}$ ), $14.6 \%$ were overweight (BMI 25-29.9 kg. $\mathrm{m}^{2}$ ) and $6.3 \%$ were obese $\left(B M I \geq 30.0 \mathrm{~kg} \cdot \mathrm{m}^{2}\right)$. Pre-pregnancy obesity and overweight were significantly associated with elevated fasting glucose $(\mathrm{p}=0.02)$, triglycerides $(p=0.01)$, and uric acid $(p=0.001)$ levels, and with decreased protective HDL cholesterol $(p=0.01)$ during pregnancy. Also, in comparison with normal weight and underweight women, obese pregnant women more often gave birth to heavier $(p=$ $0.01)$ and longer babies $(\mathrm{p}=0.036)$ and there was a strong correlation between higher pre-pregnancy BMI and delivery mode $(\mathrm{p}=$ 0.004). Obese women had $50 \%$ chance of giving birth by caesarean section in comparison with $20.1 \%$ in normal weight women. Our study confirmed that high maternal pre-pregnancy BMI could be a significant risk factor for both mother's health during pregnancy and different adverse birth outcomes, such as elevated risk of caesarean section delivery and child macrosomia. Therefore, women should be advised to attain normal weight before starting the pregnancy.
\end{abstract}

\section{Conflict of Interest}

There is no conflict of interest. 